\title{
El disfrute como mediador de la salud en el ejercicio físico*
}

\section{Enjoyment as a mediator of health in physical exercise}

Recibido: | Revisado: 26 de marzo de 2013 | Aceptado: 21 de septiembre de 2015

\author{
JuAn Antonio MoReno-MurCia ** \\ EdUARdo CERVELLÓ GIMENO *** \\ ELISA HUÉSCAR HERNÁNDEZ **** \\ ANA BELÉN AvILÉS \\ Universidad Miguel Hernández De Elche
}

doi:10.11144/Javerianaupsy15-1-dmse

Para citar este artículo: Moreno-Murcia, J.A, Cervelló, E., Huéscar, E., \& Avilés, A. (2016). El disfrute como mediador de la salud en el ejercicio físico. Universitas Psychologica, 15(1) 153-164. http:// dx.doi.org/10.11144/Javerianaupsy15-1-dmse

* Artículo de investigación.

** Catedrático de Universidad catedrático de universidad. Departamento psicología de la salud. Correo electrónico: j.moreno@umh.es

**** Catedrático de universidad. Director grupo investigación Gicom. Departamento psicología de la salud. Correo electrónico: ecervello@umh.es

****** Profesora ayudante Depto. Psicología de la Salud. Correo electrónico: ehuescar@umh.es

\section{RES UMEN}

El estudio comprobó un modelo predictivo del apoyo social de autonomía del practicante sobre la relación con los demás, a su vez sobre la motivación intrínseca; está sobre el disfrute, y por último, se comprobó la predicción del disfrute sobre el motivo fitness/salud. Se utilizó una muestra de 682 practicantes de actividades físicas no competitivas con una edad media de $35.50 \pm 11.70$ años. Los resultados del modelo de ecuaciones estructurales mostraron una predicción positiva del apoyo a la autonomía del practicante hacia la relación con los demás, esta predijo la motivación intrínseca, que a su vez predijo el disfrute y finalmente el motivo de fitness/salud. Se discuten los resultados en pro de valorar la importancia de fomentar el disfrute en los practicantes para incrementar la práctica física saludable.

Palabras clave

motivación; autonomía; salud; diversión; actividad física

\section{A B S T R A C T}

This study tested a predictive model of perception of supportive autonomy coaching on the relatedness mediator, this mediator on intrinsic motivation, intrinsic motivation on enjoyment and enjoyment on the fitness / health motive. A sample of 682 practitioners of non-competitive physical activities with a mean age of $35.50 \pm 11.70$ years, participated in the study. The results of structural equation model showed a positive prediction of supportive autonomy coaching on the relatedness mediator, relatedness on intrinsic motivation, intrinsic motivation on enjoyment and enjoyment on the fitness/health motive with $51 \%$ of variance. These results do appreciate the importance of promoting the enjoyment on practitioners to enhance the healthy physical practice.

Keywords

intrinsic motivation; autonomy; fitness/health; fun; exercise 


\section{Introducción}

Los motivos iniciales para participar en programas de ejercicio físico están muy relacionados con la motivación intrínseca, particularmente con el disfrute y la competencia (Núñez, Martín-Albo, Paredes, Rodríguez, \& Chipana, 2011; Ryan, Frederick, Lepes, Rubio \& Sheldon, 1997) pues como indican Vallerand, Deci y Ryan (1987), el compromiso deportivo es un punto importante para que las personas participen en el disfrute. En este sentido, los motivos de práctica de ejercicio físico que más se reiteran en la bibliografía especializada son la diversión y la salud (Cecchini, Méndez, \& Muñiz, 2002; Weinberg et al., 2000). Estudios previos señalan los efectos positivos de la sensación de disfrute en la realización de la práctica de actividad físico-deportiva relacionándolo con variables como la autonomía del practicante (Ryan \& Deci, 2006) o el compromiso deportivo (Ulrich-French \& Smith, 2009). Sin embargo, pese a la importancia señalada en algunos trabajos del papel de los iguales en el bienestar psicológico del practicante y en el disfrute de la actividad (Martín-Albo, Núñez, Navarro, \& Grijalvo, 2009) no existe ningún trabajo publicado hasta el momento que analice el comportamiento de estas variables de modo conjunto y estudie su influencia sobre el motivo salud de la persona.

La Teoría de la Autodeterminación (Deci \& Ryan, 2000) propone que el comportamiento es motivado por la necesidad de satisfacer tres necesidades psicológicas básicas: autonomía, competencia y relación con los demás. Las actividades que conducen a la persona a experimentar la satisfacción de las necesidades básicas son intrínsecamente gratificantes y es probable que se realicen de nuevo (Pelletier, Fortier, Vallerand, \& Brière, 2001). Por ello, una mayor percepción de las necesidades está asociada a la motivación intrínseca (Deci \& Ryan, 2000). Niveles altos de motivación intrínseca se asocian con el apoyo a la autonomía por parte del técnico (Amorose \& Anderson-Butcher, 2009; Ryan \& Deci, 2006). Varios trabajos han estudiado esta relación (Conroy \& Coasworth, 2007; Edmunds, Ntoumanis, \& Duda, 2006; Hagger et al., 2007; Moreno-Murcia, \& Sánchez-Latorre, 2016) concluyendo que el apoyo a la autonomía parece ser un componente importante y deseable del clima motivacional en el ejercicio físico. Otros estudios (Adie, Duda, \& Ntoumanis, 2008; Mageau \& Vallerand, 2003; Smith, Ntoumanis \& Duda, 2007) afirman que el apoyo a la autonomía creado por el técnico predice la satisfacción de la necesidad de relación con los demás. Conroy y Coatsworth (2007) proponen como creadores del clima del apoyo a la autonomía por parte del entrenador o técnico, el interés y la valoración, ya que están correlacionados y se relacionan negativamente con el control. Huéscar y Moreno-Murcia (2012) han señalado que proporcionar al practicante un feed-back positivo y del conocimiento del rendimiento predecía positivamente la autonomía percibida en estudiantes de educación física. Además, la percepción del apoyo a la autonomía creado por educadores eleva los niveles de autonomía hacia la tarea, el compromiso, el disfrute y el bienestar (Mageau \& Vallerand, 2003).

Las necesidades básicas juegan un papel principal en el desarrollo de la motivación intrínseca en el ejercicio físico (Barbeau, Sweet, \& Fortier, 2009; Moreno-Murcia, Huéscar \& Cervelló, 2012). En los ambientes donde el clima motivacional creado por los compañeros, orientado a la tarea, satisface las tres necesidades psicológicas básicas, los practicantes se sienten intrínsecamente motivados por la actividad, y lo hacen por la sensación de disfrute y bienestar que la implica (Moreno, López, Martínez, Alonso, \& González-Cutre, 2008). Por ello, parece particularmente importante generar climas motivacionales destinados a satisfacer las necesidades de autonomía, competencia y de relación (Amorose \& Anderson-Butcher, 2009). Numerosos trabajos han estudiado la relación entre las necesidades psicológicas y la motivación autodeterminada (Edmunds et al., 2006; Grouzet, Vallerand, Thill, \& Provencher, 2004). Aunque la mayoría de los estudios están centrados en la necesidad de competencia y/o de autonomía, cada vez se encuentran más estudios enfocados en la necesidad de relación con los demás (Losier \& Vallerand, 1995; Moreno, Conte, Borges, \& González-Cutre, 2008), donde concluyen que existe una relación positiva entre la motivación intrínseca y la necesidad de relación. La necesidad 
de relación es también uno de los motivos que lleva a los practicantes a realizar o comprometerse en actividades deportivas (García-Ferrando, 2006), debido quizás a los efectos positivos que tiene sobre la motivación intrínseca (Losier \& Vallerand, 1995; Moreno, Conte et al., 2008) y a la sensación de bienestar que provoca (Deci \& Ryan, 2000), aunque no todos los estudios están de acuerdo con esta última afirmación (Reis, Sheldon, Gable, Roscoe, \& Ryan, 2000; Sheldon \& Bettencourt, 2002).

La motivación intrínseca es uno de los factores que está asociado con el disfrute (Moreno, López et al., 2008; Reeve \& Deci, 1996; Ryan \& Deci, 2000b; entre otros). Dentro del contexto físicodeportivo, la satisfacción de las necesidades psicológicas puede conducir a un estado de motivación intrínseca, caracterizado por la búsqueda del disfrute durante la práctica (Moreno, Conte et al., 2008). En este sentido, algunos estudios (Vlachopoulos \& Karageorghis, 2005; Vlachopoulos \& Michailidou, 2006), concluyen que los perfiles motivacionales más autodeterminados se corresponden con un mayor disfrute en el ejercicio.

En el contexto del ejercicio, se ha dado importancia a las formas de motivación más autónomas y a la adherencia al ejercicio para la "ganancia de salud" (Ryan et al., 1997; Vallerand \& Losier, 1999). Diener y Emmons (1984) y Emmons (1991) proponen el disfrute/diversión como un ítem indicador de sentimiento positivo relacionado con el bienestar. Sin embargo, los estudios encontrados no relacionan el disfrute y la salud, sino entre las necesidades psicológicas básicas, el apoyo a la autonomía, motivación intrínseca y un amplio abanico de indicadores positivos relacionados con la salud, como el bienestar psicológico y físico (Castillo, Duda, Álvarez, Mercé, \& Balaguer, 2011; La Guardia \& Ryan, 2007; Wilson, Longley, Muon, Rodgers, \& Murray, 2006), la salud mental (Ryan \& Deci, 2000b), la vitalidad (Adie et al., 2008; Balaguer, Castillo, Duda, \& García-Merita, 2011; Patrick, Knee, Canevello, \& Lonsbary, 2007; Wilson et al., 2006) y la felicidad (Ryan, Huta \& Deci, 2008; Sheldon \& Niemiec, 2006) entre otros. La mayoría de estos estudios están enfocados al concepto de bienestar, siendo muy pocos los que lo relacionan con la salud, por lo que el presente estudio ha tenido como objetivo analizar las relaciones del apoyo a la autonomía creado por el técnico, la relación con los demás, la motivación intrínseca, el disfrute, y el motivo fitness/salud, en una muestra de practicantes de actividades físicas no competitivas. Partiendo de los trabajos previos, se establece como hipótesis que el apoyo a la autonomía creado por el técnico mediante la valoración y el interés, satisfará la necesidad de relación con los demás, esta a su vez predecirá la motivación intrínseca y finalmente el disfrute, que actuará como mediador positivo de la práctica física saludable.

\section{Método}

\section{Participantes}

La muestra estuvo compuesta por 682 (254 hombres y 428 mujeres) practicantes de actividades físicas no competitivas (musculación, fitness acuático, aeróbic, spinning, natación, etc.), pertenecientes al área metropolitana de una ciudad española, de edades comprendidas entre los 18 y 72 años $(M=$ 35.50, DT $=11.70$ ).

\section{Instrumentos}

Apoyo a la autonomía. Se empleó la traducción al castellano y adaptación al contexto de ejercicio físico saludable del Autonomy-Supportive Coaching Questionnaire de Conroy y Coatsworth (2007) en su versión española (Conde et al., 2010). Este cuestionario está compuesto por un total de nueve ítems agrupados en dos dimensiones: cinco ítems que miden el interés en la opinión del practicante (e.g. "mi técnico me permite elegir sobre lo que hacemos en la práctica") y cuatro ítems que evalúan la valoración del comportamiento autónomo (e.g. "mi técnico me valora por las cosas que elijo hacer en la práctica"). El cuestionario está encabezado por la frase "En mis prácticas...", respondiéndose mediante una escala de respuesta tipo Likert de 1 (totalmente en desacuerdo) a 7 (totalmente de acuerdo). El alfa de Cronbach obtenido fue de 0.82 para el factor interés en la opinión del practican- 
te y 0.80 para la valoración del comportamiento autónomo.

Relación con los demás. Se utilizó el factor relación con los demás de la versión en español (Sánchez \& Núñez, 2007) de la Escala de las Necesidades Psicológicas Básicas en el Ejercicio (BPNES) de Vlachopoulos y Michailidou (2006). El factor consta de cuatro ítems: (e.g. "me siento muy cómodo/a cuando hago ejercicio con los demás participantes"). La sentencia previa fue "En tu programa de ejercicio físico...". Las respuestas fueron puntuadas con una escala tipo Likert que oscilaba entre 1 (totalmente en desacuerdo) y 5 (totalmente de acuerdo). La consistencia interna fue de 0.89 .

Motivación intrínseca. Se emplearon los cuatro ítems (e.g. "porque me resulta placentero y satisfactorio el hacer ejercicio") del factor regulación intrínseca de la forma más actualizada en la versión traducida al castellano por Moreno, Cervelló y Martínez Camacho (2007a), de la escala Behavioral Regulation in Exercise Questionnaire-2 (Markland $\&$ Tobin, 2004). La sentencia previa fue "Yo hago ejercicio...”. Se puntúa en una escala tipo Likert que oscila de 1 (nada verdadero para mí) a 5 (totalmente verdadero para mí). El alfa obtenido fue de 0.78 .

Disfrute. Se empleó el factor disfrute de la escala de Medición de los Motivos de Actividad Física Revisada (MPAM-R) de Ryan et al. (1997), validada al contexto español por Moreno, Cervelló, y Martínez Camacho (2007b). Le precedía la frase "Realizo ejercicio físico...”. El factor está compuesto por siete ítems (e.g "porque disfruto con esta actividad") y las respuestas se responden en una escala tipo Likert de siete puntos, donde 1 corresponde a nada verdadero para mí y 7 totalmente verdadero para mí. La consistencia interna presentada por la dimensión fue de 0.81 .

Fitness/Salud. Se empleó el factor fitness/salud de la escala de Medición de los Motivos de Actividad Física Revisada (MPAM-R) de Ryan et al. (1997), validada al contexto español por Moreno et al. (2007b). Le precedía la frase "Realizo ejercicio físico...”. El factor estaba compuesto por cinco ítems (e.g "porque quiero mantener mi salud física y mi bienestar") y las respuestas respondían a una escala tipo Likert de siete puntos, donde 1 correspondía a nada verdadero para mí y 7 totalmente verdadero para mí. El alfa de Cronbach obtenido fue de 0.89.

\section{Procedimiento}

Para la recogida de la información nos pusimos en contacto con los responsables de los locales deportivos elegidos (gimnasios, piscinas, centros deportivos, etc.), para informarles de nuestros objetivos y pedirles su colaboración. Posteriormente, hablamos con los técnicos para proceder a explicarles nuestras intenciones y los beneficios que se podrían obtener de los resultados hallados, y establecer una fecha para administrar los cuestionarios a los practicantes. Se llevó a cabo la administración de los cuestionarios, estando presente el investigador principal, para hacer una breve explicación del objetivo de estudio, incrementando así el interés de los practicantes, e informar sobre cómo diligenciar el cuestionario, insistiendo en el anonimato y voluntariedad de las respuestas y en que se contestara con sinceridad, leyendo todos los ítems. El tiempo requerido para cumplimentar los cuestionarios fue de aproximadamente 15 minutos.

\section{Análisis de datos}

En primer lugar se calcularon los estadísticos descriptivos y los coeficientes de correlación entre las diferentes variables. Para calcular el poder de predicción del apoyo a la autonomía del técnico sobre el practicante, la relación con los demás, la motivación intrínseca sobre el motivo de disfrute y este sobre el de salud/fitness, se realizó un modelo de ecuaciones estructurales para analizar las relaciones hipotetizadas entre dichas variables. Los diferentes análisis se llevaron a cabo con el paquete estadístico SPSS y AMOS 19.0.

\section{Resultados}

\section{Análisis descriptivos y de correlación}

Las puntuaciones medias del apoyo a la autonomía fueron más altas en valoración que en interés. En la relación con los demás se obtuvo una media de 
4.22 y en la motivación intrínseca de 4.37 (Tabla 1). Las puntuación del motivo disfrute fue de 5.68 y de 6.20 en el fitness/salud. En el análisis de correlación se observó que todas las variables se relacionaban de forma positiva y significativa entre sí, exceptuando la dimensión interés con el motivo fitness/salud.

\section{Modelo de medición}

Para llevar a cabo el análisis, de tal forma que se mantuvieran unos grados de libertad razonables, se parcelaron los ítems de cada factor en dos grupos homogéneos, cuyas medias se utilizaron como indicadores. El modelo de medición constó de seis constructos latentes compuestos por dos variables cada uno. La dimensión valoración y el motivo fitness/salud se agruparon en dos grupos de tres y dos ítems respectivamente. La dimensión interés, relación con los demás y motivación intrínseca se parcelaron en dos grupos con dos ítems cada uno. El motivo disfrute se agrupó en dos grupos de cuatro y tres ítems (Figura 1). El análisis se realizó con el método de estimación de máxima verosimilitud. El coeficiente de Mardia fue 45.45, lo que indicaba falta de normalidad multivariada de los datos si tenemos en cuenta que este coeficiente debía ser inferior al resultado de la fórmula $p(p+2)$, donde $p$ era el número de variables observadas (Bollen, 1989). Para comprobar la bondad o semejanza del modelo teórico propuesto con los datos empíricos existentes se consideraron una serie de coeficientes fit. Así, partiendo de las aportaciones de diferentes autores
(Bentler, 1990; McDonald \& Marsh, 1990; Mulaik et al., 1989), los índices de bondad de ajuste que se consideraron para evaluar la bondad del modelo de ecuaciones estructurales fueron: $\chi^{2}, \chi^{2} /$ g.l., RMSEA (Root Mean Square Error of Aproximation), SRMR (Standardized Root Mean Square Residual) y los índices incrementales (CFI, IFI y TLI).

Estos índices de bondad de ajuste son considerados aceptables cuando el $\chi^{2} / g$.l. es inferior a 5 (Bentler, 1990), los índices incrementales (CFI, IFI y TLI) son superiores a 0.90 y los índices de error (RMSEA y SRMR) son inferiores a 0.05 y 0.08 , respectivamente. Los índices fit o índices de bondad de ajuste obtenidos $\left[\chi^{2}(39, N=682)=269.09, p=0.00, \chi^{2}\right.$ /g.l. $=6.90, \mathrm{CFI}=0.96$, IFI $=0.96$, TLI $=0.93$, SRMR $=0.05$, RMSEA $=0.08)]$, se ajustaban a los parámetros establecidos, por lo que se consideró aceptado el modelo de medición.

\section{Análisis de ecuaciones estructurales}

Los índices fit o índices de bondad de ajuste obteni$\operatorname{dos}\left[\chi^{2}(30, N=682)=310.13, p=.00, \chi^{2} / g .1 .=\right.$ 6.46, CFI $=0.95, \mathrm{IFI}=0.95, \mathrm{TLI}=0.93, \mathrm{SRMR}=$ 0.05 , RMSEA $=0.08$ )], se ajustaban a los parámetros establecidos, por lo que se consideró aceptado el modelo propuesto. Los resultados del análisis del modelo de ecuaciones estructurales (Figura 2) revelaron que el apoyo a la autonomía medido por los constructos valoración e interés predecía positivamente la necesidad psicológica básica de relación con los demás. La relación con los demás predecía positivamente la motivación intrínseca y esta a su

TABLA 1

Estadísticos descriptivos y correlaciones de todas las variables

\begin{tabular}{lccccccccc}
\hline & $\mathrm{M}$ & $\mathrm{DT}$ & $\mathrm{a}$ & 1 & 2 & 3 & 4 & 5 & 6 \\
\hline 1. Interés & 4.13 & 1.46 & 0.82 & - & $0.81^{* *}$ & $0.23^{* *}$ & $0.17^{* *}$ & $0.19^{* *}$ & 0.04 \\
2. Valoración & 4.41 & 1.37 & 0.80 & - & - & $0.32 * *$ & $0.21^{* *}$ & $0.21^{* *}$ & $0.09 *$ \\
3. Relación con los demás & 4.22 & 0.73 & 0.89 & - & - & - & $0.51^{* *}$ & $0.43^{* *}$ & $0.29 * *$ \\
4. Motivación intrínseca & 4.37 & 0.81 & 0.78 & - & - & - & - & $0.71^{* *}$ & $0.41^{* *}$ \\
5. Disfrute & 5.68 & 1.07 & 0.81 & - & - & - & - & - & $0.65^{* *}$ \\
6. Fitness/salud & 6.20 & 0.88 & 0.89 & - & - & - & - & - & - \\
\hline
\end{tabular}

Nota. $* p<0.05 ; * p<0.01$

Fuente: elaboración propia 


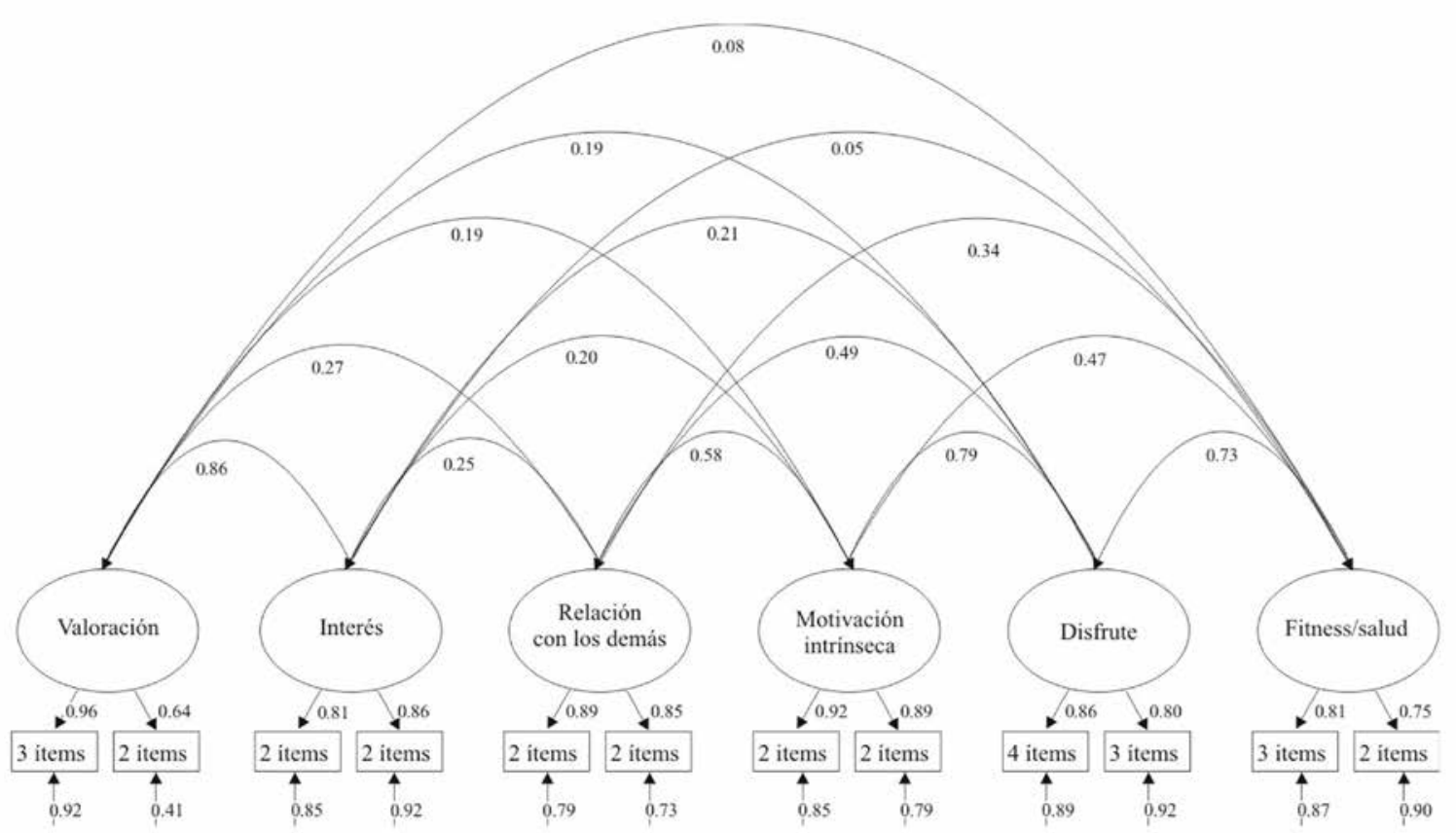

Figura 1. Modelo de medición de la estructura de seis factores hipotetizados.

Figura 1. Los círculos representan los constructos latentes y los cuadrados representan las variables medidas (grupos aleatorios de ítems). Todos los parámetros están estandarizados y son estadísticamente significativos. Las varianzas se muestran sobre las flechas pequeñas.

Fuente: elaboración propia

vez el disfrute. También el disfrute predijo positivamente el motivo fitness/salud, con una varianza total del $51 \%$.

\section{Discusión}

El estudio analizó, siguiendo el modelo Jerárquico de la Motivación Intrínseca y Extrínseca de Vallerand (2001), las relaciones entre el apoyo a la autonomía creado por el técnico, la relación con los demás y la motivación intrínseca sobre el disfrute, y este sobre el motivo fitness/salud. La novedad del estudio se encuentra en la inclusión como desencadenante del apoyo a la autonomía en el practicante de actividades físicas no competitivas, y del disfrute como mediador del motivo salud/fitness.
Los resultados del modelo de ecuaciones estructurales señalaron que el apoyo a la autonomía creado por el técnico, mediante la muestra de interés y valoración sobre la opinión y preferencias del practicante, predecía la satisfacción de la necesidad de relación con los demás, como se obtuvo en otros estudios (Adie et al., 2008; Mageau \& Vallerand, 2003; Smith et al., 2007). La bibliografía es extensa a la hora de establecer relaciones entre las necesidades psicológicas básicas y las formas de motivación más autodeterminadas (Álvarez, Balaguer, Castillo, \& Duda, 2009; Edmunds et al., 2006; Moreno, Conte et al., 2008; Vlachopoulos \& Michailidou, 2006), así como con resultados adaptativos para la persona (Soric, 2009). Tal como sucede en este estudio, la relación con los demás predecía de forma positiva la motivación intrínseca, en consonancia 


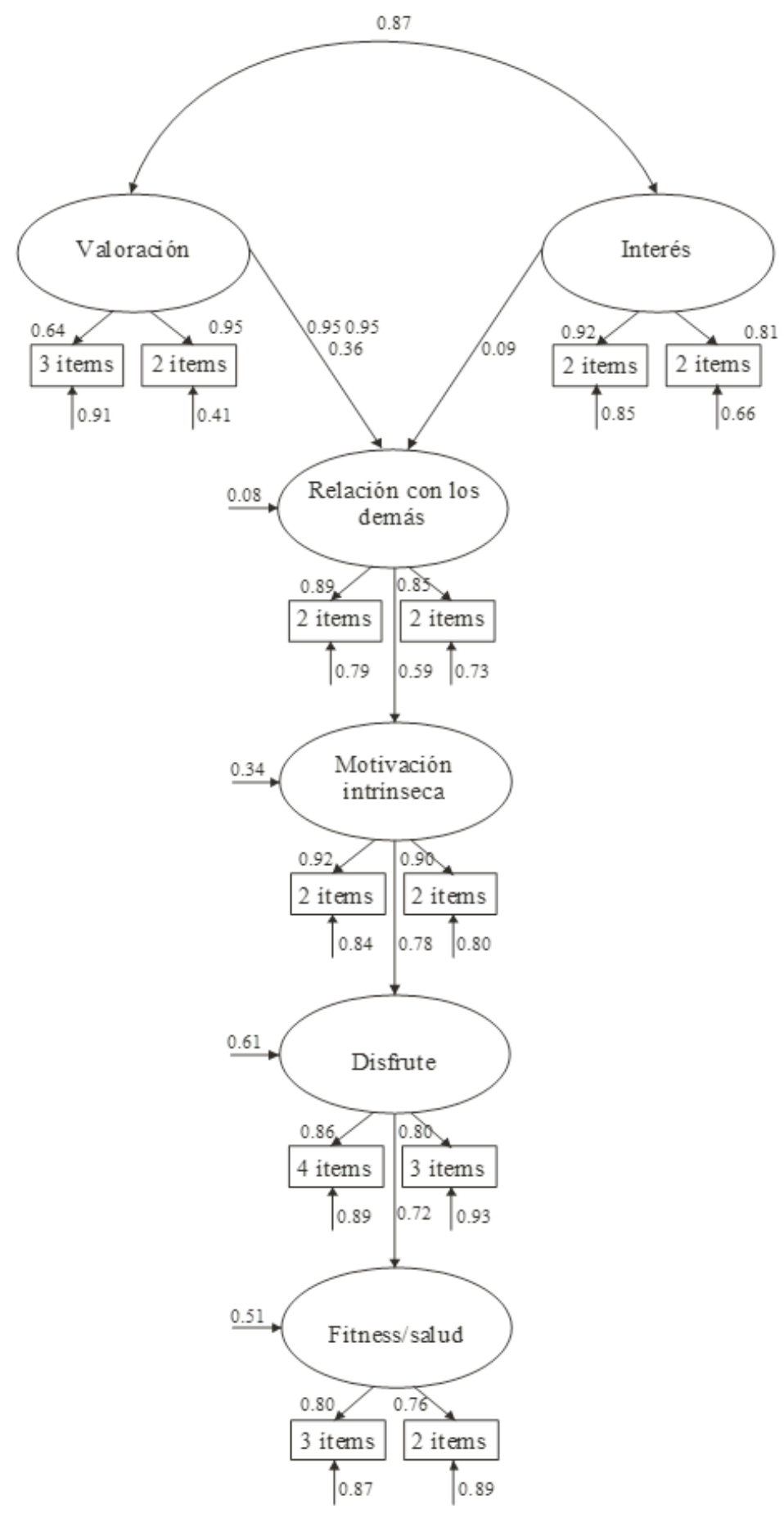

Figura 2. Modelo de ecuaciones estructurales mostrando las relaciones entre el apoyo a la autonomía, la relación con los demás, la motivación intrínseca, la diversión y el motivo de fitness/salud. Todos los parámetros están estandarizados y son estadísticamente significativos. Las varianzas se muestran sobre las flechas pequeñas.

Fuente: elaboración propia 
con lo obtenido en otras investigaciones centradas en esta necesidad psicológica (Losier \& Vallerand, 1995; Moreno, Conte et al., 2008). Por tanto, el apoyo a la autonomía puede ser la base a la hora de crear un clima adecuado que a través de la relación con los demás fomente la motivación intrínseca.

Por todo ello puede ser fundamental que los técnicos deportivos planifiquen sus intervenciones tratando de fomentar la autonomía y la relación entre los practicantes, para que estos estén intrínsecamente motivados y sean más propensos a vivenciar experiencias satisfactorias (Chatzisarantis \& Hagger, 2009; Moraes, Corte-Real, Dias, \& Fonseca, 2009). En la misma línea, Mageau y Vallerand (2003) proponen las siguientes condiciones para lograr un estilo interpersonal del técnico a favor de la autonomía: posibilitar la elección pero con reglas específicas y limitadas, justificar las tareas y los límites de las mismas, reconocer los sentimientos y la perspectiva de otras personas, proporcionar a los practicantes oportunidades de tomar iniciativas y trabajar de forma independiente, administrar un feedback positivo, evitando el feedback de control, y evitar comportamientos de control.

Tal como se hipotetizaba, y coincidiendo con estudios previos (Álvarez et al., 2009; Moreno, López et al., 2008; Shafizadeh, 2007; Vlachopoulos \& Karageorghis, 2005), la motivación intrínseca predijo positivamente el disfrute. En el modelo también se halló que el disfrute actuó como predictor del motivo fitness/salud. Los estudios encontrados no muestran relaciones directas entre disfrute y salud, sino entre las necesidades psicológicas básicas, la motivación intrínseca, el apoyo a la autonomía y el bienestar (Adie et al., 2008; Milyavskaya \& Koestner, 2011; Reinboth \& Duda, 2006; Wilson et al., 2006), pudiéndose encontrar conexiones con el disfrute con las consecuencias positivas obtenidas de las relaciones establecidas.

Este estudio da un paso más en la relación entre el disfrute y la actividad física saludable, mostrando que el disfrute puede ayudar a promover la práctica física saludable. Weinberg et al. (2000) afirman que en la mayoría de estudios con personas adultas los principales motivos para participar suelen ser los relacionados con la salud. Es de vital importancia que el practicante disfrute en las actividades físicas para fomentar la práctica y la adherencia a la actividad, ya que diversos trabajos muestran que existe una relación entre el disfrute y el compromiso deportivo (Ryan et al., 1997; Torregrosa, Sousa, Viladrich, Villamarín, \& Cruz, 2008; Vlachopoulos \& Karageorghis, 2005). Por lo tanto, disfrutar en la práctica puede ayudar a fomentar el compromiso con esta, y sentir que realmente se está mejorando la salud. Del mismo modo, otros estudios podrían seguir analizando las relaciones entre el disfrute y el motivo fitness/salud en contextos de ejercicio físico no competitivo, para tratar de establecer relaciones causales y dar explicación a los resultados expuestos en este trabajo. Ya que el estado de flow es ideal para estar intrínsecamente motivado durante la práctica (Kimiecik, 2000), y guarda una estrecha relación con la motivación intrínseca, las necesidades psicológicas básicas (Kowal \& Fortier, 1999) y con la diversión (Jackon, 1996), sería interesante profundizar en estas conexiones en futuras investigaciones buscando la relación con la promoción de la práctica física saludable. Por tanto, establecer nuevas relaciones junto con el concepto del compromiso deportivo del practicante (Almagro, Sáenz-López \& Moreno, 2010), ya que es muy posible que exista una relación positiva entre estos conceptos, siguiendo la línea de pensamiento de Jackson (1996), Kimiecik (2000) y Moreno, Conte et al. (2008) donde es probable comprometerse con la práctica para volver a vivenciar experiencias positivas y satisfactorias.

Este trabajo no permite establecer relaciones causa-efecto dado su carácter correlacional y transversal. Sin embargo, el desarrollo de un modelo teórico que relaciona las dimensiones estudiadas supone un paso más para el avance del conocimiento de los procesos motivacionales en el ejercicio físico. Sería conveniente que nuevas investigaciones abordaran el estudio de estas relaciones de una forma experimental. Por ejemplo, se podrían plantear intervenciones a nivel situacional por parte del técnico tratando de dar información encaminada a la adquisición de autonomía en el practicante y ver su efecto en la motivación y satisfacción con la práctica. También, debido al problema de modelos equivalentes que presenta la técnica de ecuaciones 
estructurales (Hershberger, 2006) se asume que el modelo planteado en este estudio no sería más que uno de los posibles.

Este estudio aporta información relevante sobre como el disfrute actúa como mediador en la práctica de actividades físicas saludables. Los resultados muestran que el apoyo a la autonomía junto con la satisfacción de la necesidad psicológica básica de relación con los demás predijo positivamente la motivación intrínseca, y esta a su vez el disfrute. El disfrute también mostró ser un predictor del motivo fitness/salud. Ya que el disfrute está relacionado positivamente con la práctica física saludable, parece adecuado que los técnicos propicien el disfrute en las actividades físicas organizadas, buscando que los practicantes experimenten altos niveles de satisfacción, pues posiblemente esta situación pueda hacer que la persona siga practicando para volver a vivenciar esta experiencia.

\section{Referencias}

Adie, J., Duda, J., \& Ntoumanis, N. (2008). Autonomy support, basic need satisfaction and the optimal functioning of adult male and female sport participants: a test of basic needs theory. Motivation and Emotion, 32, 189-199.

Almagro, B. J., Sáenz-López, P., \& Moreno, J. A. (2010). Prediction of sport adherence through the influence of autonomy-supportive coaching among Spanish adolescent athletes. Journal of Sports Science and Medicine, 9, 8-14.

Álvarez, M. S., Balaguer, I., Castillo, I., \& Duda, J. L. (2009). Coach autonomy support and quality of sport engagement in young soccer players. The Spanish Journal of Psychology, 12, 138-148.

Amorose, A. J., \& Anderson-Butcher, D. (2009). Autonomy-supportive coaching and self-determinated motivation in high school and college athletes: A test of self-determination theory. Psychology of Sport and Exercise, 8(5), 654-670.

Balaguer, I., Castillo, I., Duda, J. L., \& García-Merita, M. (2011). Asociaciones entre la percepción del clima motivacional creado por el entrenador, orientaciones disposicionales de meta, regulaciones motivacionales y vitalidad subjetiva en jóvenes jugadoras de tenis. Revista de Psicología del deporte, 20(1), 133-148.

Barbeau, A., Sweet, S., \& Fortier, M. (2009). A pathanalytic model of self-determination theory in a physical activity context. Journal of Applied Biobehavioral Research, 14(3), 103-118.

Bentler, P. M. (1990). Comparative fit indexes in structural models. Psychological Bulletin, 107, 238-246.

Bollen, K. A. (1989). Structural equations with latent variables. New York: Wiley.

Castillo, I., Duda, J. L., Álvarez, M. S., Mercé, J., \& Balaguer, I. (2011). Clima motivacional, metas de logro de aproximación y evitación y bienestar en futbolistas cadetes. Revista de Psicología del Deporte. 20(1), 149-164.

Cecchini, J. A., Méndez, A., \& Muñiz, J. (2002). Motives for practicing sport in Spanish schoolchildren. Psicothema, 14, 523-531.

Chatzisarantis, N., \& Hagger, M. (2009). Effects of an intervention based on self-determination theory on self-reported leisure-time physical activity participation. Psychology $\mathcal{E}$ Health, 24(1), 29-48.

Conde, C., Sáenz-López, Carmona, J., González-Cutre, D., Martínez Galindo, C., \& Moreno, J. A. (2010). Validación del Cuestionario de Percepción de Apoyo a la la Autonomía en el Proceso de Entrenamiento (ASCQ) en jóvenes deportistas españoles. Estudios de Psicología, 31(2), 145-157.

Conroy, D. E., \& Coasworth, J. D. (2007). Assesssing autonomy-supportive coaching strategies in youth sport. Psychology of Sport and Exercise, 8, 671-684.

Deci, E. L., \& Ryan, R. M. (2000). The "what" and "why" of goal pursuits: human needs and the selfdetermination of behaviour. Psychological Inquiry, 11, 227-268.

Diener, E., \& Emmons, R. (1984). The independence of positive and negative affect. Journal of Personality and Social Psychology, 47, 105-1117.

Edmunds, J., Ntoumanis, N., \& Duda, J. L. (2006). A test of Self-Determination Theory in the exercise domain. Journal of Applied Social Psychology, 36, 2240-2265.

Emmons, R. (1991). Personal strivings, daily life events, and psychological and physical well-being. Journal of Personality, 59, 453-472. 
García-Ferrando, M. (2006). Posmodernidad y deporte: Entre la individualización y la masificación. Encuesta sobre hábitos deportivos de los españoles 2005. Madrid: CSD y CIS.

Grouzet, F., Vallerand, R., Thill, E., \& Provencher, P. (2004). From environmental factors to outcomes: a test of an integrated motivational sequence. Motivation and Emotion, 28, 331-346.

Hagger, M. S., Chatzisarantis, N. L. D., Hein, V., Pihu, M., Soós, I., \& Karsai, I. (2007). The perceived autonomy support scale for exercise settings (PASSES): development, validity, and cross-cultural invariance in young people. Psychology of Sport and Exercise, 8, 632-653.

Hershberger, S. L. (2006). The problem of equivalent structural models. En G. R. Hancock y R. O. Mueller (Eds.), Structural equation modeling: a second course (pp. 13-42). Greenwich, CT: Information Age Publishing.

Huéscar, E., \& Moreno-Murcia, J. A. (2012). Relación del tipo de feed-back del docente con la percepción de autonomía del alumnado en clases de educación física. Infancia y Aprendizaje, 35(1), 87-98.

Jackson, S. (1996). Toward a conceptual understanding of the flow experience in elite athletes. Research Quarterly for Exercise and Sport, 67, 76-90.

Kimiecik, J. C. (2000). Learn to love exercise. Psychology Today, 33, 20-22.

Kowal, J., \& Fortier, M. S. (1999). Motivational determinants of flow contributions from Self Determination Theory. The Journal of Social Psychology, 139, 355-368.

La Guardia, J. G., \& Ryan, R. M. (2007). Why identities fluctuate: variability in traits as a function of situational variations in autonomy support. Journal of Personality, 75, 1205-1228.

Mageau, G. A., \& Vallerand, R. J. (2003). The coachathlete relationship: a motivational model. Journal of Sport Sciences, 21, 883-904.

Markland, D., \& Tobin, V. (2004). A modification to the Behavioural Regulation in Exercise Questionnaire to include an assessment of a motivation. Journal of Sport and Exercise Psychology, 26, 191-196.

Martín-Albo, J., Núñez, J. L., Navarro, G., \& Grijalvo, F. (2009). Un modelo motivacional explicativo del bienestar psicológico en la universidad. Revista de Psicología del Deporte, 26(1), 41-54.

McDonald, R. P., \& Marsh, H. W. (1990). Choosing a multivariate model: non centrality and goodness of fit. Psychological Bulletin, 107, 247-255.

Milyavskaya, M., \& Koestner, R. (2011). Psychological needs, motivation, and well-being: a test of self-determination theory across multiple domains. Personality and Individual Differences, 50(3), 387-391.

Moraes, M., Corte-Real, N., Dias, C., \& Fonseca, A. M. (2009). Satisfaçao com a vida, excercicio físico e consume de Tabaco en adolescentes de diferentes áreas geográficas de Portugal. Revista Brasileña de Ciencias del Deporte, 30(2), 137-149.

Moreno, J. A., Cervelló, E. M., \& Martínez Camacho, A. (2007a). Measuring self-determination motivation in a physical fitness setting: validation of the Behavioral Regulation in Exercise Questionnaire-2 (BREQ-2) in a Spanish sample. The Journal of Sport Medicine and Physical Fitness, 47(3), 366-378.

Moreno, J. A., Cervelló, E., \& Martínez Camacho, A. (2007b). Validación de la Escala de Medida de los Motivos para la Actividad Física-Revisada en españoles: diferencias por motivos de participación. Anales de Psicología, 23, 167-176.

Moreno, J. A., Conte, L., Borges, F., \& González-Cutre, D. (2008). Necesidades psicológicas básicas, motivación intrínseca y propensión a la experiencia autotélica en el ejercicio físico. Revista Mexicana de Psicología, 25, 305-312.

Moreno, J. A., López, M., Martínez, C., Alonso, N., \& González-Cutre, D. (2008). Peer's influence on exercise enjoyment: a self-determination theory approach. Journal of Sports Science and Medicine, 7, 23-31.

Moreno-Murcia, J. A., Huéscar, E., \& Cervelló, E. (2012). Prediction of adolescents doing physical activity after completing secondary education. The Spanish Journal of Psychology, 15(1), 90-100.

Moreno-Murcia, J. A., \& Sánchez-Latorre, F. (2016). The effects of autonomy support in physical education classes. RICYDE, Revista Internacional de Ciencias del Deporte, 12(43), 79-89.

Mulaik, S. A., James, L. R., Van Astine, J., Bennett, N., Lind, S., \& Stilwell, C. D. (1989). Evaluation 
of goodness-of-fit indices for structural equation models. Psychological Bulletin, 105, 430-445.

Núñez, J. L., Martín-Albo, J., Paredes, A., Rodríguez, O., \& Chipana, N. (2011). The mediating role of perceived competence testing a motivational sequence in university students. Universitas Psychologica, 10(3), 669-680.

Patrick, H., Knee, C. R., Canevello, A., \& Lonsbary, C. (2007). The role of need fulfillment in relationship functioning and well-being: a self-determination theory perpective. Journal of Personality and Social Psychology, 92, 434-457.

Pelletier, L. G., Fortier, M. S., Vallerand, R. J., \& Brière, N. M. (2001). Associations among perceived autonomy support, forms of self-regulation, and persistance: a prospective study. Motivation and Emotion, 25, 279-306.

Reeve, J., \& Deci, E. L. (1996). Elements of the competitive situation that affect intrinsic motivation. Personality and Social Psychology Bulletin, 6, 228-233.

Reinboth, M., \& Duda, J. L. (2006). Perceived motivational climate, need satisfaction and indices of well-being in team sports: a longitudinal study. Psychology of Sport and Exercise, 7, 269-286.

Reis, H. T., Sheldon, K. M., Gable, S. L., Roscoe, J., \& Ryan, R. M. (2000). Dialy well-being: the role of autonomy, competence, and relatedness. Personality and Social Psychology Bulletin, 26, 419-435.

Ryan, R. M., \& Deci, E. L. (2000b). Self-determination theory and the facilitation of intrinsic motivation, social development, and well-being. American Psychologist, 55, 68-78.

Ryan, R. M., \& Deci, E. L. (2006). Self-regulation and the problem of human autonomy: Does psychology need choice, self-determination, and will? Journal of Personality, 74(6), 1557-1585.

Ryan, R. M., Frederick, C. M., Lepes, D., Rubio, N., \& Sheldon, K. M. (1997). Intrinsic motivation and exercise adherence. International Journal of Sport Psychology, 28, 335-354.

Ryan, R. M., Huta, V., \& Deci, E. L. (2008). Livingwell: a self-determination theory perpective on eudaimonia. Journal of Happiness Studies, 9, 139-170.

Sánchez, J. M., \& Núñez, J. L. (2007). Análisis preliminar de las propiedades psicométricas de la versión española de la Escala de Necesidades Psicológicas
Básicas en el Ejercicio Físico. Revista Iberoamericana de Psicología del Ejercicio y el Deporte, 2(2), 83-92.

Shafizadeh, M. (2007). Relationships between goal orientation, motivational climate and perceived ability with intrinsic motivation and performance in physical education university students. Journal of Applied Sciences, 7, 2866-2870.

Sheldon, K. M., \& Bettencourt, B. A. (2002). Psychological need-satisfaction and subjective well-being within social groups. British Journal of Social Psychology, 41, 25-38.

Sheldon, K., \& Niemiec, C. (2006). It's not just the amount that counts: balanced need satisfaction also affects well-being. Journal of Personality and Social Psychology, 91, 331-341.

Smith, A. L., Ntoumanis, N., \& Duda, J. L. (2007). Goal striving, goal attainment, and well-being: an adaptation and testing of the self-concondance model in sport. Journal of Sport and Exercise Psychology, 29, 763-782.

Soric, I. (2009). Regulatory styles, causal attributions and academic achievement. School Psychology International, 30 (4), 403-420.

Torregrosa, M., Sousa, C., Viladrich, C., Villamarín, F., \& Cruz, J. (2008). El clima motivacional y el estilo de comunicación del entrenador como predictores del compromiso en futbolistas jóvenes. Psicothema, 20, 254-259.

Ulrich-French, S., \& Smith, A. L. (2009). Social and motivational predictors of continued youth sport participation. Psychology of Sport and Excercise, 10, 87-95.

Vallerand, R. J. (2001). A hierarchical model of intrinsic and extrinsic motivation in sport and exercise. En G. C. Roberts (Ed.), Advances in motivation in sport and exercise (pp. 263-319). Champaign, IL: Human Kinetics.

Vallerand, R. J., Deci, E. L., \& Ryan, R. M. (1987). Intrinsic motivation in sport. Exercise and Sport Sciences Reviews, 15, 389-425.

Vallerand, R. J., \& Losier, G. F. (1999). An integrative analysis of intrinsic and extrinsic motivation in sport. Journal of Applied Sport Psychology, 11, 142-169. 


\section{Juan Antonio Moreno-Murcia, Eduardo Cervelló Gimeno, Elisa Huéscar Hernández, Ana BelÉn Avilés}

Vlachopoulos, S., \& Karageorghis, C. (2005). Interaction of external, introyected, and identified regulation with motivation in exercise: relationships with exercise enjoyment. Journal of Applied Biobehavioral Research, 20, 113-132.

Vlachopoulos, S., \& Michailidou, S. (2006). Development and initial validation of a measure of autonomy, competence and relatedness in exercise: the basic psychological needs in exercise scale. Measurement in Physical Education and Exercise Science, 10, 179-201.
Weinberg, R., Tenenbaum, G., McKenzie, A., Jackson, S., Anshel, M., Grove, R., \& Fogarty, G. (2000). Motivation for youth participation in sport and physical activity: relationships to culture, selfreported activity levels, and gender. International Journal of Sport Psychology, 31, 231-346.

Wilson, P., Longley, K., Muon, S., Rodgers, W., \& Murray, T. C. (2006). Examining the contributions of perceived psychological need satisfaction to wellbeing in exercise. Journal of Applied Biobehavioral Research, 11, 243-264. 